\title{
ABORDAGEM DO TABAGISMO EM UMA SALA DE ESPERA: UMA EXPERIÊNCIA EXTENSIONISTA
}

\author{
Luiz Eduardo de Almeida \\ Universidade Federal de Juiz de Fora \\ luiz.almeida@ufff.edu.br \\ Marília Nalon Pereira \\ Universidade Federal de Juiz de Fora \\ marilia.nalon@ufff.edu.br
}

\author{
Valéria de Oliveira \\ Universidade Federal de Juiz de Fora \\ valeria.oliveira@ufff.edu.br \\ Diego Machado de Oliveira \\ Universidade Federal de Juiz de Fora \\ diego-oliveira2608@hotmail.com \\ Larisse Matins Aguiar \\ Universidade Federal de Juiz de Fora \\ laa.risse@hotmail.com
}

Resumo

Objetivo: o presente estudo, através de um relato, materializa experimentações vivenciadas pelo "Projeto de Extensão Sala de Espera" (PESE). Método: direcionada pela demanda do serviço local, coube à equipe extensionista, norteada pela temática "Tabagismo", o desenvolvimento de uma ação de cunho educativo e preventivo em uma sala de espera. Utilizando-se do instrumento "TPC", as ações foram dinamizadas em três tempos sequenciados: "O pensar", "O fažer" e "O refletir". Resultados: ponderando-se as forças e as fragilidades encontradas, o grupo de trabalho consagrou a experiência como exitosa. Conclusão: o trabalho reforça o fundamental papel na extensão na formação profissional, a efetividade do instrumento "TPC", o reconhecimento da sala de espera como espaço coletivo para a promoção de atividades educativas, incluindo aí a dinamização da temática "Tabagismo" e, por fim, desperta a importância de valorizar a divulgação das vivências extensionistas nos espaços científicos. Período: de março a abril de 2016.

Palavras-chave: Sala de Espera. Educação em Saúde. Tabagismo.

\section{APPROACH TO SMOKING IN A WAITING ROOM: AN EXTENSIONIST EXPERIENCE}

\begin{abstract}
Objective: the present study, through an account, materializes experiments experienced by the "Extension Project Waiting Room" (EPWR). Method: guided by the demand of the local service, it was the extension team, guided by the theme "Tobacco use disorder", the development of an educational and preventive action in a waiting room. Using the "TPC" instrument, the actions were dynamized in three sequenced tempos: "To think", "To do" and "To reflect". Results: by considering the strengths and weaknesses found, the work group recognized the experience as successful. Conclusion: the study reinforces the fundamental role of extension in vocational training, the effectiveness of the "TPC" instrument, the recognition of the waiting room as a collective space for the promotion of educational activities, including the promotion of "Tobacco use disorder" end, it awakens the importance of valorizing the dissemination of extensionist experiences in scientific spaces. Period: from March to April 2016.
\end{abstract}

Keywords: Waiting Room. Health Education. Tobacco Use Disorder.

\section{ENFOQUE DEL TABAQUISMO EN UNA SALA DE ESPERA: UNA EXPERIENCIA EXTENSIONISTA}

\begin{abstract}
Resumen
Objetivo: el presente estudio, a través de un relato, materializa experimentaciones vivenciadas por el "Proyecto de Extensión Sala de Espera" (PESE). Método: dirigida por la demanda del servicio local, correspondió al equipo extensionista, orientada por la temática "Tabaquismo", el desarrollo de una acción de cuño educativo y preventivo en una sala de espera. Utilizando el instrumento "TPC", las acciones fueron dinamizadas en tres tiempos secuenciados: "El pensar", "El hacer" y "El reflejar". Resultados: ponderando las fuerzas y las fragilidades encontradas, el grupo de trabajo consagró la experiencia como exitosa. Conclusión: el trabajo refuerza el fundamental papel en la extensión en la formación profesional, la efectividad del instrumento "TPC", el reconocimiento de la sala de espera como espacio colectivo para la promoción de actividades educativas, incluyendo allí la dinamización de la temática "Tabaquismo" finalmente, despierta la importancia de valorizar la divulgación de las vivencias extensionistas en los espacios científicos. Período: de marzo a abril de 2016.
\end{abstract}

Palavras clave: Sala de Espera. Educación en Salud.Tabaquismo. 
Abordagem do tabagismo em uma sala de espera: uma experiência extensionista

\section{INTRODUÇÃO}

$\mathrm{Na}$ intenção de reforçar o cenário extensionista do Campus Avançado de Governador Valadares da Universidade Federal de Juiz de Fora, UFJF/GV surge, em 2014, o "Projeto de Extensão Sala de Espera”, PESE. Como referenda seu nome, em seu desenho metodológico, prevêse o desenvolvimento de ações multiprofissionais, de cunho educativo-preventivas, junto a usuários em momento de espera em Unidades de Atenção Primária à Saúde, UAPS, no município de Governador Valadares-MG.

No tocante aos ambientes de espera, a sistemática do PESE vai de encontro ao firmado em diversos estudos, que reconhecem nestes ambientes, se dinamizados, cenários propícios para o desenvolvimento de programas de educação em saúde. Dentro desta perspectiva, a sala de espera se consubstancia em um espaço que permite inserir novos conceitos, tirar dúvidas e, principalmente, criar vínculos com os usuários, em síntese, encerra-se em um fundamental lugar de acolhimento e humanização (ALMEIDA et al., 2017; ALMEIDA, ANDRADE, ZAKARON, 2016; NORA, MÂNICA, GERMANI, 2009; PAIXÃO, CASTRO, 2006; RODRIGUES et al., 2009; TEIXEIRA, VELOSO, 2006; ROSA, BARTH, GERMANI, 2011; SILVA et al., 2013; VALENTE et al., 2015).

Quanto à multiprofissionalidade, composto por quinze integrantes, o PESE fortalece a referida lógica de trabalho, evidenciando cinco enfoques formativos (Farmácia, Fisioterapia, Medicina, Nutrição e Odontologia), uma estruturação que consoa ao dito nos trabalhos Almeida, Pereira, Oliveira (2016), Ribeiro, Pires e Blank (2004) e Peduzzi (2001). Os autores afirmam que, nos cenários de atenção em saúde, trabalhar em equipe de modo integrado significa conectar diferentes processos de trabalho, com base no conhecimento do trabalho do outro e valorização da participação deste na produção de cuidados, encerrando-se, portanto, em uma prestação de serviços mais contextualizada e humanizada (ALMEIDA, PEREIRA, OLIVEIRA, 2016; RIBEIRO, PIRES, BLANK, 2004; PEDUZZI, 2001).

Neste movimento, a extensão universitária não apenas pluraliza cenários práticos para uma formação mais direcionada às necessidades do Sistema Único de Saúde, SUS, bem como atua no provimento da tão almejada articulação entre "ensino" (PESE-UFJF/GV), "serviço" (Prefeitura Municipal de Governador Valadares, PMGV, contexto prático) e "comunidade" (usuários da UAPS do bairro Esperança).

Uma lógica de trabalho corroborante ao dito por Almeida, Pereira, Oliveira (2016), Albuquerque et al. (2008) e Madeira (2006), que reconhecem nos cenários extensionistas um importante território acadêmico para a concepção do enlace ensino-serviço-comunidade. Os 
Abordagem do tabagismo em uma sala de espera: uma experiência extensionista

autores também destacam o fundamental papel desta articulação junto à reorientação da formação dos profissionais de saúde, afinal, são nos espaços da extensão que se dão a socialização do resultado de um fato (pesquisa) e/ou de um aprendizado (ensino).

De outra forma, avigorada aos preceitos de Freire (2006 a,b; 2007), a extensão universitária se define aos moldes da "via de mão dupla", ou seja, além de levar informações para a comunidade, traz para o cenário universitário dados e informações, coletados e interpretados cientificamente, que retratam as complexidades da integralidade da vida humana (FREIRE, 2006a, 2006b, 2007).

Nesta enseada todas as ações desenvolvidas pelo PESE-UFJF/GV foram pactualizadas com as demandas do serviço local, creditando nesta sistemática o provimento de ações mais contextualizadas e direcionadas às reais necessidades da população adstrita. Não obstante, em março de 2016, foi designado pelos enfermeiros gestores da UAPS assistida, a abordagem da temática "Tabagismo" juntos aos usuários em espera.

Cabe destacar que a pertinência do assunto levantado encontra ampla justificativa na literatura científica. Afinal, segundo Nunes e Castro (2011), o tabagismo se constitui o maior fator de morte evitável no mundo, portanto, risco para dezenas de doenças e agravos. Indo além, as consequências do hábito de fumar não se encerram nos fumantes, pelo contrário, afetam a saúde coletiva. Assim, do ponto de vista de políticas públicas, pelos impactos nos custos governamentais, trata-se de um problema de saúde pública. Os autores ainda destacam a necessidade de se prover espaços educativos de discussão sobre assunto, não alimentados na perseguição dos fumantes, pelo contrário, alicerçados no apoio ao complexo processo de cessação de fumar (NUNES, CASTRO, 2011).

Por fim, permeado ao engenho supradescrito, o presente estudo se propõe, através de um relato de experiência, prover um recorte analítico das experimentações vivenciadas no PESEUFJF/GV. Assim, vislumbrou-se neste trabalho uma oportunidade de ofertar a outros leitores um momento de autoanálise, afinal, muitos podem se identificar com determinados aspectos, situações e reflexões.

\section{DESENVOLVIMENTO}

Em acordo com a Resolução de n 510 , de 07 de abril de 201617, o presente trabalho foi avaliado e liberado pelo Comitê de Ética da Universidade Federal de Juiz de Fora, conforme parecer de número 2.056.630, emitido em 10 de maio de 2017 (BRASIL, 2016). 
Abordagem do tabagismo em uma sala de espera: uma experiência extensionista

Trata-se de um relato de experiência, qualitativamente estruturado sob estratégia narrativo-descritiva e moldado à técnica argumentativa.

Por fim, sob recorte transversal, evidenciam-se a coleta e a análise de dados. O primeiro momento foi instrumentalizado pelo "Relatórios de atividades do PESE-UFJF/GV: Tabagismo em foco", que, além de informações textuais, trazem em seus anexos a transcrição de áudios e arquivos fotográficos. Já a exploração analítica das informações, segundo Minayo et al (1994), p. 24, “Os autores que seguem tal corrente não se preocupam em quantificar, mas, sim, em compreender e explicar a dinâmica das relações sociais [...] desse ponto de vista, a linguagem, as práticas e as coisas são inseparáveis”.

\section{RESULTADOS E ANÁLISES}

Frente à sua iminente demanda, a de desenvolver na sala de espera da UAPS do bairro Esperança (Governador Valadares, MG) uma atividade de cunho educativo-preventiva, abarcando a temática tabagismo, a equipe extensionista se via diante de uma importante questão: "Como desenvolver?".

Atravessado por esta questão, o grupo elegeu como guia o dispositivo “TPC” (Imagem 1), que segundo seus idealizadores, Almeida, Pereira, Bara (2009) e Almeida, Pereira, Oliveira (2016),

"O instrumento apresentado [...] se desenvolve em três etapas: Teorizando ("o pensar"), Praticando ("o fazer") e Criticando ("o refletir"), sendo por isso denominado TPC. Sistematicamente, as etapas se complementam, trazendo em seu bojo conceitual a relação entre planejamento estratégico com a eficácia, eficiência e efetividade de ações de educação em saúde" (ALMEIRA, PEREIRA, OLIVEIRA, p.746, 2016).

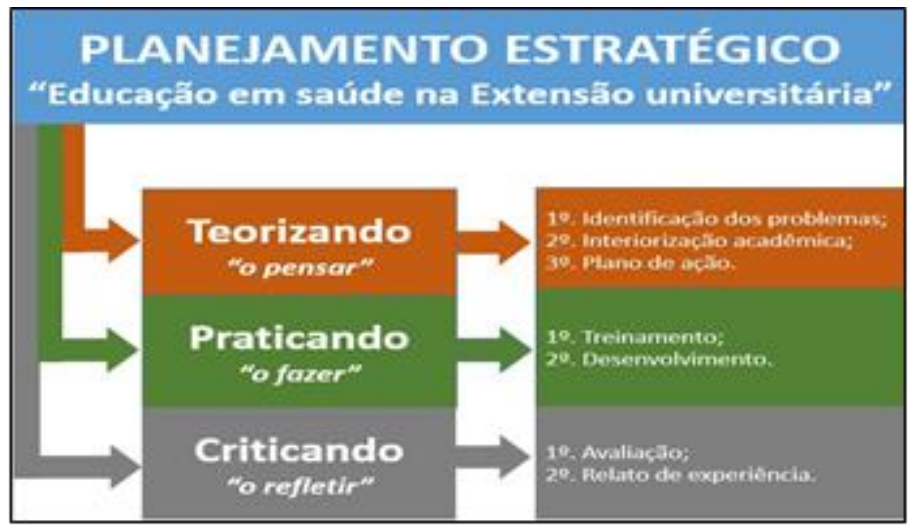

Imagem 1 - Instrumento "TPC".

Fonte: Almeida, Pereira, Oliveira, p.746, 2016

Aqui cabem as reflexões de Almeida, Pereira e Bara (2009), p. 29, que não veem no "TPC" uma "fórmula mágica", pelo contrário, ele traz como intuito a aproximação, de forma crítica 
Abordagem do tabagismo em uma sala de espera: uma experiência extensionista

e reflexiva, dos envolvidos junto a seu futuro cenário de atuação, afinal, de acordo com os mesmos autores, a extensão é dinamizada pela rica lógica do "ensinar a fazer contextualizado".

A partir de então, interfaceando-se a demanda com o dispositivo pedagógico, sequenciam-se três etapas: 1.Teorizando/ "O pensar" (14/03 e 21/03/2016); 2.Praticando/ "O fazer" (29/03, 30/03 e 01/04/2016) e 3.Criticando/ "O refletir" (de 04/04/2016).

Antevendo o primeiro encontro, mais precisamente com uma semana de antecedência, a coordenação docente do projeto solicitou aos acadêmicos extensionistas uma coleta crítica e reflexiva de informações sobre "Tabagismo". Neste ensejo, para melhor orientar os discentes envolvidos, forneceu-se um questionário, sendo ele constituído por três perguntas problematizadoras (1. “O que é?”; 2. “Como percebo no meu corpo/espaco?”; 3. “Como prevenir/tratar?”).

No dia 14/03/2016, iniciada pela apresentação dos direcionados dados levantados pelos acadêmicos, partiu-se, após ampla discussão, para o alinhamento dos enfoques multiprofissionais. Posteriormente, de posse dos conteúdos refletidos, levantou-se uma inquietação para o próximo encontro: "Como levar as informações discutidas para o nosso cenário prático?".

Assim, em 21/03/2016, permeados pela interpelação, dentro das prerrogativas preconizadas pela metodologia "Brainstorming", foi construído o "Planejamento Estratégico da Ação". Daqui, em linhas gerais, delineou-se a sistemática da ação programada, alimentada por duas atividades, uma palestra e uma dinâmica.

A primeira atividade foi fundamentada na discussão de duas questões chaves, "o fumante" e "a ajuda". Neste momento, desdobrariam-se diversas argumentações, como o hábito físico e comportamental do fumante, a dificuldade em se desligar do vício, a importância em procurar e aceitar ajuda, as substâncias nocivas presentes no cigarro, a desintoxicação com o decorrer do tempo naqueles que param de fumar e, principalmente, onde encontrar serviços de apoio para abandonar o tabagismo. Dinamizadas por materiais didáticos ("Painel" e "panfletos"), os conteúdos trabalhados não se encerrariam na palestra, visto os apetrechos não apenas ficarem afixados no quadro de aviso da UAPS ("Cantinho da UFJF-GV'), bem como serem direcionados aos Agentes Comunitários de Saúde, ACS, da UAPS, desta forma, as informações ali contidas ficariam livres para carreamento, sejam para usuários em diferentes momentos de espera, bem como no decorrer de visitas domiciliares.

Continuando, na intenção de analisar o aprendizado e a apreensão, delineou-se a dinâmica "Vencer o tabagismo junto é mais fácil". A atividade proposta consistiria na distribuição de balas aos usuários em espera, tão logo, seria a eles solicitado que tentassem abrir a embalagem usando apenas uma das mãos. Após algum tempo, e sem entenderem o propósito da dinâmica, muitos sentiriam a dificuldade e, até mesmo, não conseguiriam executar o desafio a eles direcionado. 
Abordagem do tabagismo em uma sala de espera: uma experiência extensionista

Durante as tentativas, levantar-se-ia o questionamento: "Quem pensou em pedir ajuda a quem está do lado?". A atividade se centra na intenção de mostrar que muitos conseguem atingir o objetivo da brincadeira, entretanto, fica claro que buscar ajuda junto às pessoas mais próximas em muito facilitaria o processo. Em síntese, uma analogia ao vivido pelo tabagista, que deveria procurar ajuda para concretizar o tão almejado abandono do fumo.

Encerrado o módulo teórico, parte-se para a segunda etapa, Praticando/ "O fazer", didaticamente dividida em dois momentos: "Treinamento" e "Desenvolvimento".

Não obstante, no dia 29/03/2016, os discentes do PESE, junto a seu corpo docente, apresentaram e aprovaram seu planejamento estratégico. Este momento vai de encontro ao dito por Almeida e Oliveira Jr. (2009), p. 64, "treinar não é eximir-se do erro, pelo contrário, no treino, através da mimetização de uma realidade, vislumbra-se capacitar uma equipe em prover estratégias secundárias para se contornar os tão diversos e frequentes obstáculos da vida real".

Assim, previamente treinados, os membros do PESE-UFJF/GV partiram para o "Desenvolvimento" das atividades programadas. Buscando ampliar uma maior cobertura populacional, com demanda média de 35 usuários por dia, a prática se deu em dois encontros, 30/03 e 01/04/2016 (Imagem 2).

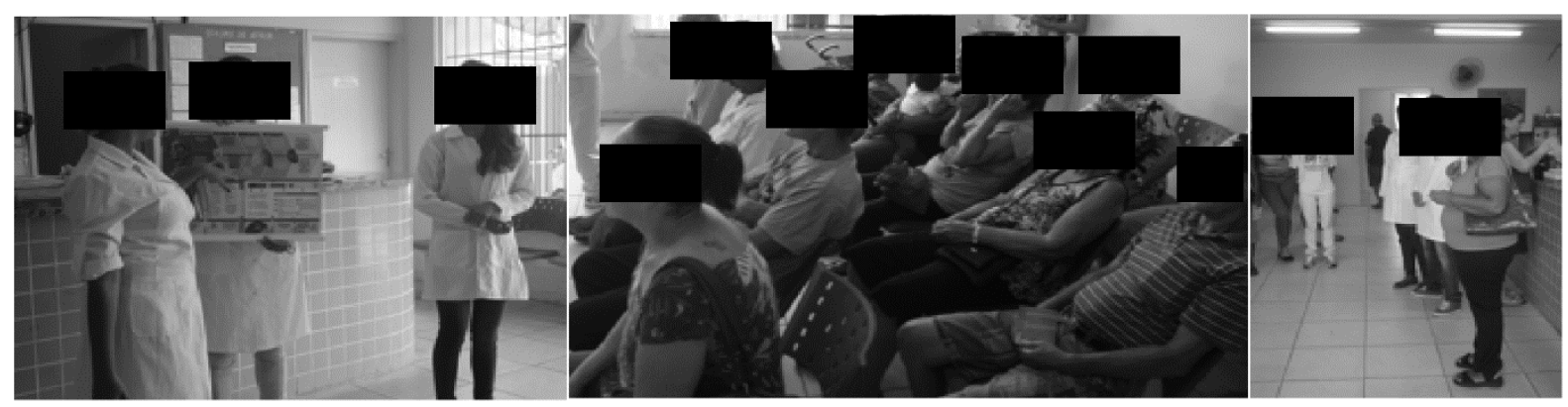

Imagem 2 - Sala de espera - UAPS-Esperança, Governador Valadares, MG. Fonte: Relatórios de atividades do PESE-UFJF/GV: “Tabagismo em foco”, 2016

Partindo das experimentações vivenciadas, no tocante à palestra, ficou evidenciada sua aceitação, tanto pelos pacientes quanto pelos profissionais ali presentes. Apesar de ser amplamente difundido por diversas mídias, quando feita a abertura para a participação da comunidade, que, além de dúvidas e comentários, reforçaram a qualidade da equipe do PESE ao abordarem o tema de forma diferenciada, atuando em conjunto com o público ali presente e levantando alguns pontos ainda desconhecidos por muitos ("Quais são e como atuam as substâncias nocivas presentes no cigarro", "Como ocorre a desintoxicação com o decorrer do tempo naqueles que param de fumar" " "Serviços de apoio e tratamento na UAPS"). 
Abordagem do tabagismo em uma sala de espera: uma experiência extensionista

No tocante à atividade lúdica, pôde-se perceber um maior despertar quanto à atenção e participação dos espectadores, portanto, reforçando a dificuldade de se combater o tabagismo, o qual está instalado em nossa sociedade por décadas, e principalmente, a importância em aceitar e oferecer ajuda àqueles que lutam contra tal vício.

Enfim, quanto ao carreamento das informações trabalhadas, refoça-se o papel continuado dos materiais didáticos produzidos. Além das esperadas aplicabilidades (afixados na UAPS e direcionados para as atividades de visita domiciliares), os profissionais da unidade evidenciaram sua utilização junto a grupos operacionais.

Até aqui, pode-se afirmar que as experimentações vivenciadas contemplam o preconizado por Nunes e Castro (2011), afinal, segundo as autoras, em uma abordagem educativa com fumantes, torna-se crucial envolvê-los em atitudes agregadoras, conscientizá-los de possíveis crises de abstinência e, principalmente, direcioná-los a locais de apoio e tratamento.

Encerrando a sistemática preconizada pelo "TPC", chega a terceira etapa, Criticando/ "O refletir", aparada por dois tempos sequenciais, "Avaliaģão" e "Relato de experiência".

Assim, no dia 04/04/2016, intermediado pelos docentes coordenadores, coube à equipe discente fazer a "Avalição", de forma crítica e reflexiva, de suas experiências. Para melhor orientar os extensionistas, este momento contou com uma questão indutora: "Quais os pontos positivos e negativos percebidos durante todo o processo?'.

Dentre os pontos positivos, evidenciaram-se: "Escutamos o serviço", "Usuários receptivos e interessados", "Formação profissional", "Planejamento adequado das atividades", "Presença dos professores durante o desenvolvimento das ações", "Profissionais da UAPS interessados" e "A presença de discentes de diferentes cursos da área da saúde foi de suma importancia, pois contribuiram para explanação de diferentes pontos de vista sobre o tabagismo".

Contraponto, "Ruidos durante a apresentação que fugia do controle da equipe palestrante", "A dificuldade em avaliar a qualidade das atividades", "Alinhar os horários de aula com as atividades de extensão" e "A falta de identificação da instituição junto à comunidade - muitas pessoas não sabiam o que era UFJF-GV", foram os principais pontos negativos levantados.

Posteriormente, ponderando as reflexões supradescritas, consensualmente, o grupo extensionista consagrou as vivências como exitosa. Deste processo analítico, distinguiram que o posicionamento positivo não foi pautado exclusivamente em seus acertos, pelo contrário, reconheceram nos erros um papel fundamental para um aprendizado mais coerente com a realidade.

Indo além, em consonância com diversos estudos, pode-se afirmar que o espaço "Sala de espera” se consagra como um território dinâmico para atividades extensionistas, onde diferentes 
Abordagem do tabagismo em uma sala de espera: uma experiência extensionista

indivíduos aguardam seu atendimento de saúde, constituindo-se, portanto, em um ambiente fértil para implantação de ações educativas, que podem contribuir significativamente para a promoção da saúde, prevenção de agravos e encaminhamento para outras atividades, portanto, encorpando e otimizando ainda mais o papel da atenção primária junto aos serviços de saúde prestados pelo Sistema Único de Saúde, SUS. (ALMEIDA et al., 2017; ALMEIDA, ANDRADE, ZAKARON, 2016; NORA, MÂNICA, GERMANI, 2009; PAIXÃO, CASTRO, 2006; RODRIGUES et al., 2009; TEIXEIRA, VELOSO, 2006; ROSA, BARTH, GERMANI, 2011; SILVA et al., 2013; VALENTE et al., 2015).

Por fim, este trabalho se encerra com a última etapa de sua sistemática, a construção de seu "Relato de experiência". Corroborando com preconizado por Almeida, Pereira e Oliveira (2016), p.747,

"é fundamental que as experimentacoèes extensionistas sejam compartilhadas, reconhecendo-se na publicação cientifica um dos mais importantes instrumentos de divulgação. Entre as diversas metodologias, destaca-se o relato de experiência, ressaltando que sua construção não deve ser direcionada apenas aos acertos, ou seja, deve-se oferecer espaço também para discutir erros e fragilidades, já que uma equipe aprende com os acertos e se transforma com os erros".

\section{CONSIDERAÇÕES FINAIS}

Direcionado pelo seu objetivo, o de analisar as experimentações vivenciadas pelo PESEUFJF/GV no desenvolvimento de uma atividade educativo-preventiva, que teve o "Tabagismo" como enfoque temático, evidenciaram-se algumas conquistas, destacando:

- a ampliação das ações extensionistas da UFJF-GV, na cidade de Governador Valadares (MG), voltadas a atender às demandas da sociedade local;

- a percepção do fundamental papel da extensão na formação profissional, espaço este onde se socializa com a comunidade o aprendizado (ensino) e com a academia a experiência (pesquisa);

- a efetividade do instrumento "TPC" na dinamização das atividades extensionistas programadas;

- o reconhecimento da sala de espera como um espaço público, de solidariedade, de diálogo e de educação conscientizadora, de incentivo a transformação e ao exercício da cidadania; 
Abordagem do tabagismo em uma sala de espera: uma experiência extensionista

- a sensibilização dos usuários frente ao entendimento e ao reconhecimento de seu papel ativo como disseminadores de informações e, principalmente, da sua importância no controle ativo do "Tabagismo";

- a valorização da divulgação das vivências extensionistas nos espaços científicos.

Por fim, calcado no empoderamento de seus elementos empíricos, esta investigação não se baseou em testar hipótese, pelo contrário, galgou-se aqui a construção de um espaço argumentativo, não apenas direcionado pelos acertos encontrados, bem como fortemente alimentado pela discussão e, principalmente, pelos aprendizados advindos dos inevitáveis entraves e frustrações.

A partir de então, vislumbrou-se neste trabalho uma oportunidade de ofertar a outros leitores um momento de autoanálise, afinal, muitos podem se identificar com determinados aspectos, situações e reflexões. Nessa toada, credita-se o real papel da produção científica, o de ser um espaço contextualizado e, qualitativemnte, direcionado às solutividades das desafiantes aflições que assolam as complexidades da vida humana.

\section{REFERÊNCIAS}

ALBUQUERQUE, V.S.; GOMES, A.P.; REZENDE, C.H.A.; SAMPAIO, M.X.; DIAS, O.V.; LUGARINHO, R.M. A integração ensino-serviço no contexto dos processos de mudança na formação superior dos profissionais da saúde. Rev. bras. educ. med, v.32, n.3, p.:356-362, 2008.

ALMEIDA, L.E.; ANDRADE, L.M.D.; ZACARON, K.A.M. Sala de espera em extensão: percursos para a implantação e consolidação de um projeto multiprofissional. Caminho Aberto Revista de Extensão do IFSC, 3(4): 124-127, 2016.

ALMEIDA, L.E.; OLIVEIRA JÚNIOR, G.I. Sistema de Execução do Projeto. In: Pró-Saúde: Ensino, Pesquisa e Extensão - Almeida, L.E. et al. Juiz de Fora: Editar Juiz de Fora, 2009. pp.: 63-86.

ALMEIDA, L.E.; PEREIRA, M.N.; BARA, É.F. Programa de Capacitação de Ideias (PCI). In: Pró-Saúde: Ensino, Pesquisa e Extensão - Almeida, L.E. et al. Juiz de Fora: Editar Juiz de Fora, 2009. pp.: 165-91.

ALMEIDA, L.E.; PEREIRA, M.N.; OLIVEIRA, V. Governador Valadares (MG) em Extensão: Interfaces para a Dinamização e Instrumentalização do Cenário Extensionista em um Campus recém-Implantado. Revista Brasileira de Educação Médica, RBEM, 40(4): 743-750, 2016.

ALMEIDA, L.E.; OLIVEIRA, V.; PEREIRA, M.N.; OLIVEIRA, D.M.; AGUIAR, L.M. Sala de espera em extensão: doenças sexualmente transmissíveis em foco. Interfaces - Revista de Extensão da UFMG, 5(1): 198-205, 2017. 
Abordagem do tabagismo em uma sala de espera: uma experiência extensionista

BRASIL. Ministério da Saúde. Conselho Nacional de Saúde. Resolução nº510, de 07 de abril de 2016. Brasília: Ministério da Saúde, 2016.

FREIRE, P. Extensão ou Comunicação. Rio de Janeiro: Paz e Terra. 2006 a.

FREIRE, P. Pedagogia da Autonomia: saberes necessários à pratica educativa. Rio de Janeiro: Paz e Terra, 2006b.

FREIRE, P. Educação como Prática da Liberdade. Rio de Janeiro: Paz e Terra. 2007.

MADEIRA, M.C. Ensino, Pesquisa, Extensão. In: Carvalho, Antônio César Perri; Kriger, Léo (organizadores). Educação Odontológica. São Paulo: Editora Artes Médicas, 2006. pp.: 97-103.

MINAYO, M.C.S. Ciência, técnica e arte: o desafio da pesquisa social. In: Deslandes, Suely Ferreira (Organizadora). Pesquisa Social: teoria, método e criatividade. Rio de Janeiro: Editora Vozes, 1994. pp.: 09-29.

NORA, C.R.D.; MÂNICA, F.; GERMANI, A.R.M. Sala de espera uma ferramenta para efetivar a educação em saúde. Revista Saúde e Pesquisa, 2(3): 397-402, 2009.

NUNES, S.; CASTRO, M.R.P. Tabagismo: Abordagem, prevenção e tratamento. Londrina: EDUEL, 2011. 224 p.

PAIXÃO, N.R.A.; CASTRO, A.R.M. Grupo sala de espera: trabalho multiprofissional em Unidade Básica de Saúde. Boletim da Saúde, 20(02): 71-78, 2006.

PEDUZZI, M. Equipe multiprofissional de saúde: conceito e tipologia. Rev Saúde Pública, 35(1):103-9, 2001.

RIBEIRO, E.M.; PIRES, D.; BLANK, V.L.G. A teorização sobre o processo de trabalho em saúde como instrumental para a análise do Programa de Saúde da Família. Cad Saúde Pública, 20(2):438-446, 2004.

RODRIGUES, A.D.; DALLANORA, C.R.; ROSA, J.; GERMANI, A.R.M. Sala de espera: um ambiente para efetivar a educação em saúde. Vivências, 5(7): 101-106, 2009.

TEIXEIRA, E.R.; VELOSO, R.C. O grupo em sala de espera: território de práticas e representações em saúde. Texto Contexto Enferm, 15(2): 320-325, 2006.

ROSA, J.; BARTH, P.O.; GERMANI, A.R.M. A sala de espera no agir em saúde: espaço de educação e promoção à saúde. Perspectiva, 35(129): 121-130, 2011.

SILVA, G.G.S.; PEREIRA, E.R.; OLIVEIRA, J.O.; KODATO, Y.M. Um momento dedicado à espera e à promoção da saúde. Psicologia: Ciência e Profissão, 33(4): 1000-1013, 2013.

VALENTE, M.A.S.; ANDRADE, A.G.; ALCÂNTARA, P.G.; SILVA, P.S.A. O que te espera na Sala de Espera: educação em saúde em Unidades de Atenção Primária à Saúde (UAPS) de Governador Valadares (MG). Caminho Aberto - Revista de Extensão do IFSC, 1(3): 137-141, 2015. 